This item was submitted to Loughborough's Research Repository by the author.

Items in Figshare are protected by copyright, with all rights reserved, unless otherwise indicated.

\title{
Transmission through optically generated inductive grid arrays
}

PLEASE CITE THE PUBLISHED VERSION

http://dx.doi.org/10.1109/22.775484

PUBLISHER

(c) IEEE

VERSION

VoR (Version of Record)

LICENCE

CC BY-NC-ND 4.0

REPOSITORY RECORD

Lockyer, David S., J.C. Vardaxoglou, and Michael J. Kearney. 2019. "Transmission Through Optically Generated Inductive Grid Arrays”. figshare. https://hdl.handle.net/2134/9451. 
This item was submitted to Loughborough's Institutional Repository (https://dspace.lboro.ac.uk/) by the author and is made available under the following Creative Commons Licence conditions.

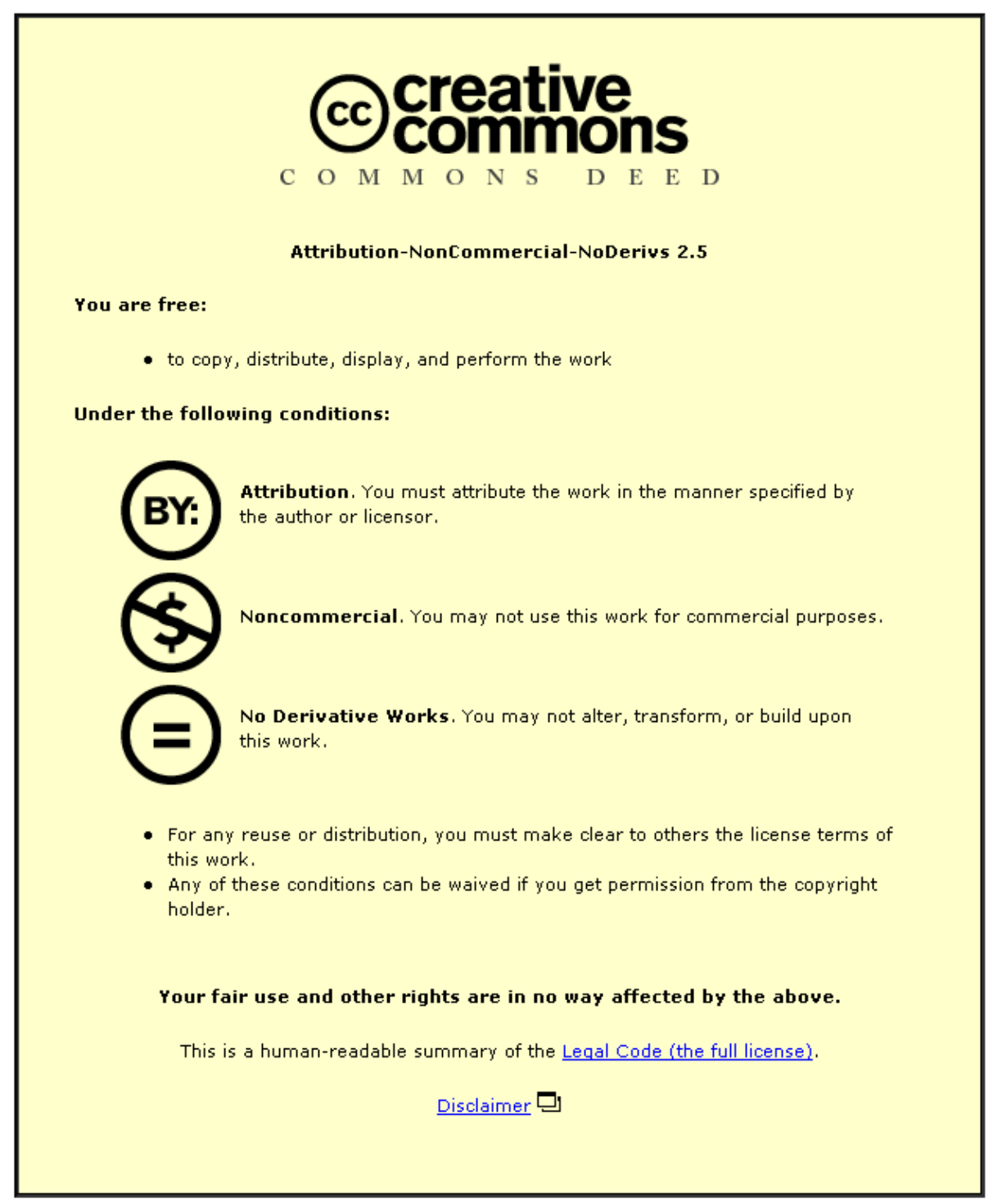

For the full text of this licence, please go to: http://creativecommons.org/licenses/by-nc-nd/2.5/ 


\title{
Transmission Through Optically Generated Inductive Grid Arrays
}

\author{
D. S. Lockyer, J. C. Vardaxoglou, Member, IEEE, and M. J. Kearney
}

\begin{abstract}
A technique is developed for generating pseudometallic plasma inductive grid arrays within a semiconductor wafer. The induced plasma elements are defined by a surface impedance which is discussed. Results of an investigation into the transmission properties of a variety of array designs between 16 and $40 \mathrm{GHz}$ are presented. Measurements are performed on a silicon wafer illuminated by an external optical source via a negative image mask.
\end{abstract}

Index Terms-Arrays, frequency selective surfaces, optical control of microwaves.

\section{INTRODUCTION}

O PTICAL control of the behavior of the substrate medium of periodic arrays and frequency selective surfaces (FSS) can be achieved by changing the microwave properties of the substrate [1]. The passband of an array of aperture dipoles printed onto silicon can be switched on or off by varying the (complex) dielectric properties of the semiconductor under photoexcitation. The photoconductive properties of silicon are such that an electron-hole pair plasma is produced, and when there is sufficient concentration, it behaves like a conductor. This property of silicon is well known and has been utilized previously in the investigation of leaky wave radiation from dielectric slab waveguides [2], [3], control of Bragg gratings in a dielectric waveguide [4], reflection properties of photoinduced strip gratings [5], optically controlling a silicon coplanar waveguide [6] and, more recently, generation of a singular antenna element element using a light tank [7]. As wafers increase in size, printing an antenna array and operating it at microwave frequencies becomes more manageable. Dynamic tuning offers additional and alternative degrees of freedom in the functionality of a system, wherein adaptive and/or reconfigurable systems could result. Tuning with the use of optical signals has attractions because of the many salient features they offer; fast switching speeds, electromagnetic compatability (EMC), integration, size and weight. A way in which tuning can be enhanced is to electronically imprint an array in the semiconductor and using a variable pattern optical source to alter its microwave response. We have shown theoretically that plasma arrays can be formed, by selectively doping the semiconductor with impurities [8], as well as in

Manuscript received October 12, 1998; revised March 10, 1999. This work was supported by a grant from the U.K. Engineering and Physical Sciences Research Council (EPSRC).

The authors are with the Department of Electrical and Electronic Engineering, Loughborough University, Loughborough, Leicestershire LE11 3TU, U.K.

Publisher Item Identifier S 0018-9480(99)05213-8.
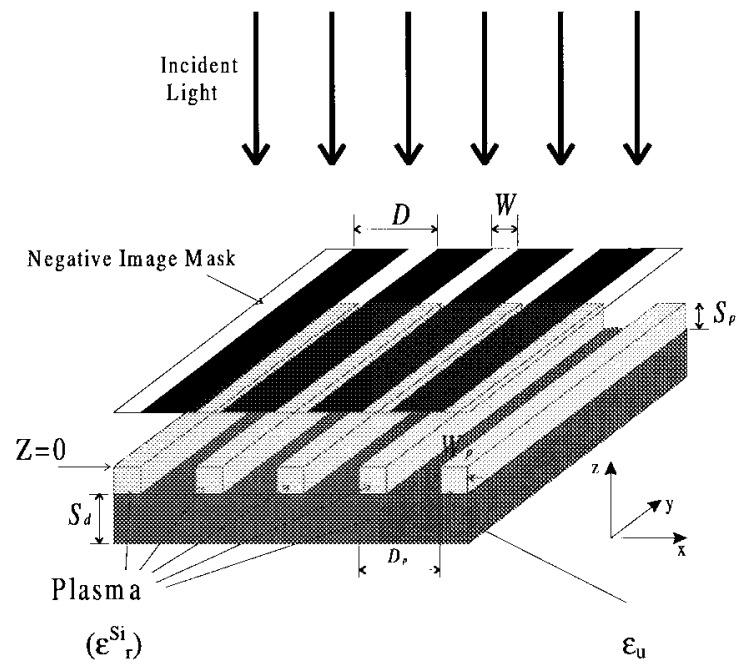

Fig. 1. Array generation using an optical mask.

a thin semiconductor layer on a supporting sapphire substrate [9].

In this paper, we generate an inductive grid array by fixing an optical mask between a light source and a single wafer, as shown in Fig. 1. A uniform light illumination will generate the plasma only under the transparent areas of the mask. By selecting different mask patterns the array dimensions can be changed. Both theoretical and experimental results are presented whereby the transmission properties are examined for a variety of array designs between 16 and $40 \mathrm{GHz}$. High resistivity, (typically $>6 \mathrm{k} \Omega \cdot \mathrm{cm}$ ) silicon has been used here as the semiconductor substrate. In the mathematical model the plasma is represented as a surface impedance of uniform thickness. An outline of the analysis steps is given together with a derivation of the surface impedance representing the plasma grid array.

\section{ANALYSIS}

Changes in the dielectric material properties can be achieved by means of an optical source of variable intensity. The underlying principle is the photoillumination of the semiconductor substrate so that electron-hole pairs are generated, thus producing a plasma. Here, we assume that the plasma thickness remains constant while the plasma density varies. Following the geometry shown in Fig. 1, the array is represented by the photoinduced plasma elements whose thickness is $S_{p}$ and complex relative permittivity $\varepsilon_{r}^{\mathrm{Si}}$, (given in the Appendix). $\varepsilon_{r}^{\mathrm{Si}}$ is a function of the microwave frequency and the plasma 
density $N$. These elements lie on the $x-y$ plane and can be characterized by the surface impedance, which varies according to $\varepsilon_{r}^{\mathrm{Si}}$. This impedance is taken into account in the integral equation analysis below. The remainder of the dielectric is unexcited (dark) silicon of thickness $S_{d}$, and relative dielectric constant $\left(\varepsilon_{u}\right.$, which is assumed to be real. The grids on the mask are oriented along $y$, and have a periodicity $D$ and a width $W$.

Here, we adopt the analysis for periodic arrays of infinite extent, where Floquet modes $\left(\boldsymbol{\Psi}_{p q}\right)$ represent the tangential electric and magnetic fields. The procedure follows that given in [10], with the exception of the inclusion of the surface impedance. The latter is derived for normal incidence only.

For propagation along the positive $z$ axis, the tangential electric and magnetic field expansions are generally formed to include transmitted and reflected waves. In the dark dielectric region, these are given as follows:

$$
\begin{aligned}
\boldsymbol{E}(\boldsymbol{r}, z)= & \sum_{m p q}\left(T_{m p q} e^{-j \beta_{p q} z}+R_{m p q} e^{+j \beta_{p q} z}\right) \boldsymbol{\Psi}_{m p q}(\boldsymbol{r}) \\
\boldsymbol{H}(\boldsymbol{r}, z)= & \sum_{m p q} \eta_{m p q}\left(T_{m p q} e^{-j \beta_{p q} z}\right. \\
& \left.-R_{m p q} e^{+j \beta_{p q} z}\right) \hat{z} \times \boldsymbol{\Psi}_{m p q}(\boldsymbol{r})
\end{aligned}
$$

where $T_{m p q}$ and $R_{m p q}$ are the modal transmission and reflection coefficients respectively. $\eta_{m p q}$ is the modal admittance, $p q$ are the modal indices and $m=1,2$ stands for TM and TE modes, respectively.

The propagation constant is

$$
\beta_{p q} \sqrt{k_{0}^{2} \varepsilon_{u}-k_{t x}^{2}-k_{t y}^{2}}
$$

where $k_{t x}$ and $k_{t y}$ are the tangential wave vector components, and $k_{0}$ is the freespace wave-number. $k_{t x}$ and $k_{t y}$ depend on the array periodicity, modal indices and angles of the plane wave incidence [10]. Applying the standard electromagnetic boundary conditions, a relationship between $T_{m p q}$ and $R_{m p q}$ is obtained:

$$
\frac{R_{m p q}}{T_{m p q}}=\left(\frac{\eta_{m p q}^{\mathrm{air}}-\eta_{m p q}}{\eta_{m p q}^{\mathrm{air}}+\eta_{m p q}}\right) e^{-2 j \beta_{p q} S_{d}}
$$

In addition, the modal reflection coefficient is expressed in terms of the spectrum of the unknown current $J$. For this derivation see [10, eq. (2.47)]. Enforcing the condition of the total (scattered and incident) electric field $\boldsymbol{E}^{\text {tot }}=Z_{s p} \boldsymbol{J}$, where $Z_{s p}$ is the surface impedance, we derive the electric field integral equation, which is given by

$$
\sum_{m p q} C_{m p q}^{\mathrm{scat}} \tilde{J}_{m p q} \boldsymbol{\Psi}_{p q}(\boldsymbol{r})-Z_{s p} J=-\sum_{m=1}^{2} C_{m o o}^{n s c} T_{m}^{\mathrm{inc}} \boldsymbol{\Psi}_{o o}(\boldsymbol{r})
$$

where $\tilde{J}$ is the Fourier transform of $J$. The right-hand side represents the incident field and the $C$ 's are terms involving the substrate parameters, [10]. This equation is reduced, using the method of moments, into a matrix form by expanding the unknown currents as a set of entire domain bases. The array is generated by the formation of the electric current $J$ as the surface impedance approaches zero. We have assumed that there are no normal currents flowing down the plasma elements. Once the currents have been estimated, the copolar transmission coefficient can then be calculated, [10]. Under a variable light intensity the skin depth varies only in the elements. At low density levels, the imaginary part of the refractive index is small, therefore, the skin depth is very large. On the other hand, as the plasma becomes more dense the skin depth decreases to values smaller than the plasma thickness. Where the impedance is zero, the array has all its elements fully conducting. As the impedance increases the surface conductance decreases and the array begins to behave in a lossy manner, and eventually at very small plasma concentrations it vanishes altogether.

Assuming that there is no diffusion into the silicon substrate and the plasma thickness is smaller than the wavelength the surface impedance can be calculated from the source-free Maxwell-Ampere equation [11]. For normal incidence

$$
Z_{s p}=\frac{E_{o}}{J_{\text {tot }}} \quad \Omega / \square
$$

where $E_{o}\left(=T_{o}+R_{o}\right)$ is the tangential electric field in the absence of scatterers $(p=q=0)$ at $z=0$. $J_{\text {tot }}$ is the total surface current per unit width of plasma in the $y$-direction and is derived from the integration of the effective current density, $J_{e d}$. $J_{e d}$ consists of both the effective conduction and effective displacement currents and is equal to the tangential magnetic field at the surface of the plasma medium [12]. Thus, in the absence of sources:

$$
J_{e d}=\sigma\left(T_{o} e^{-j \beta_{o} z}+R_{o} e^{+j \beta_{o} z}\right)
$$

where $\sigma=j \omega \varepsilon_{o} \varepsilon_{r}^{\mathrm{Si}}$.

Integrating (6) over $S_{p}$ yields $J_{\text {tot }}$. Substituting the latter into (5) and making use of (3) (in the absence of scatterers), the following equation for the plasma impedance is derived:

$$
Z_{s p}^{\mathrm{Si}}=Z_{o} \frac{\sinh \left(j k_{o} n_{r}^{\mathrm{Si}} S_{p}\right)+n_{r}^{\mathrm{Si}} \cosh \left(j k_{o} n_{r}^{\mathrm{Si}} S_{p}\right)}{n_{r}^{\mathrm{Si}}\left(\cosh \left(j k_{o} n_{r}^{\mathrm{Si}} S_{p}\right)+n_{r}^{\mathrm{Si}} \sinh \left(j k_{o} n_{r}^{\mathrm{Si}} S_{p}\right)-1\right)}
$$

where $Z_{O}$ is the impedance in vacuum and the complex refractive index $n_{r}^{\mathrm{Si}}\left(=\sqrt{\varepsilon_{r}^{\mathrm{Si}}}\right)$ is shown in Fig. 9 .

The surface impedance takes into account all possible scenarios and also covers the special cases, referred to as penetrable and impenetrable, usually considered in conventional printed element arrays [13]. The surface impedance is a function of the concentration, that exists only on the area occupied by the elements. Since the optical excitation will alter the complex refractive index of silicon, any state will be attainable, from dielectric to good conductor inclusive. Fig. 2 shows the impedance for different plasma concentrations. At low concentration, there is high negative reactance, the magnitude of which decreases as the plasma thickness increases, whereas the resistance remains small. At the merging point of the real and imaginary parts of the refractive index, both impedance parts also begin to merge. Just before that point, (near $N=10^{15} \mathrm{~cm}^{-3}$ ), the real part of the impedance reaches a maximum and the imaginary part has a steep slope. 


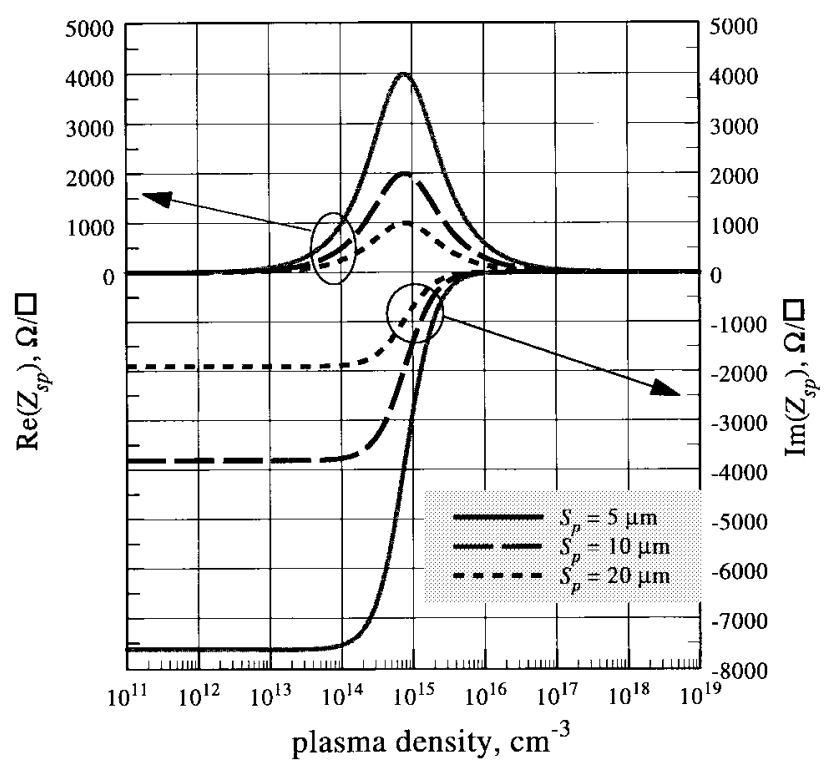

Fig. 2. Surface Impedance as a function of $N$.

As the plasma thickness increases the resistance decreases. This decrease has some bearing on the amount of plasma concentration required to generate the array, and is discussed in the results below.

\section{EXPERIMENTAL SETUP}

The array is generated on silicon wafers, which are discs of $100 \mathrm{~mm}$ diameter and thickness $330 \mu \mathrm{m}$. The discs are of a high resistivity (typically $>6 \mathrm{k} \Omega \cdot \mathrm{cm}$ ). This is a critical property of the wafers and means that in the dark state the silicon acts principally like a dielectric. A low resistivity implies more metallic behavior, which is the property we are inducing by means of the illumination, so it is important for the dark silicon to possess a high resistivity in order that its behavior contrasts with that of the generated plasma regions.

A negative image mask is used in conjunction with a Xenon strobe lamp to generate the plasma regions on the wafer (Fig. 1). When the light is incident upon the mask it penetrates only the transparent portions into the silicon thereby establishing the plasma distribution as a negative image of the mask. A Xenon source is used because it has a peak in its spectrum around $980 \mathrm{~nm}$ and the absorption coefficient of silicon is low at this wavelength while the penetration depth is high. This leads to good conditions for the plasma generation. The array image on the mask has periodicity $D$, and the strips are of width $W$. In an ideal situation the dimensions of the plasma grid would be identical to those of the mask, but for several reasons this is often not the case. Hence, the periodicity and width of the plasma strips are denoted $D_{p}$ and $W_{p}$, respectively. The light passing through the mask will experience a degree of diffraction from the pattern and diffusion in the silicon so that the actual plasma array is not likely to be sharply defined in practice, although for clarity it is shown as so in Fig. 1 where the plasma depth, $S_{p}$, represents the penetration into the silicon of the incident photons. A further remark on this point is that while assessing

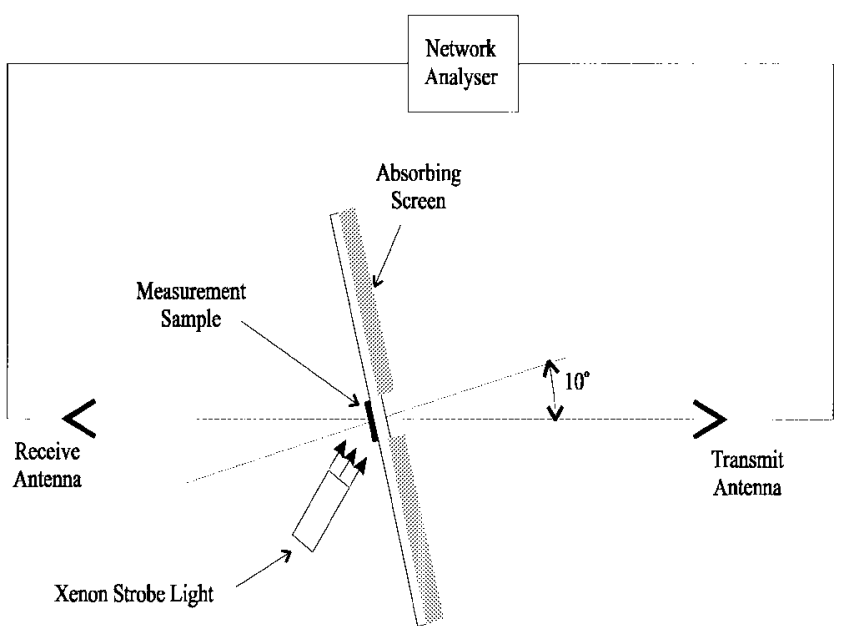

Fig. 3. Transmission measurement setup under optical illumination.

the transmission properties of the grid structures, the light cannot be normally incident on the wafer because the source will interfere with the path of the transmitted field.

Fig. 3 shows the measurement set up and highlights the issue of making transmission measurements while illuminating the wafer. The absorbing screen is rotated slightly to present an incidence angle of about $10^{\circ}$ to the electromagnetic field from the transmit antenna. Behind the screen, the lamp is placed so that the optimum trade off is reached between incident light intensity on the wafer, uniform illumination of the wafer and interference free propagation for the transmitted fields. The Xenon strobe lamp has a maximum discharge of 140J and a flash duration of about $6 \mathrm{~ms}$ with a maximum of $10 \mathrm{flash} / \mathrm{s}$.

\section{Plane-WaVe Transmission}

Results have been obtained for a variety of grid dimensions and some representative comparisons between measurement and theory are presented in this section. In all cases the mask is mounted on the wafer by using a very thin layer of a spray adhesive. The wafer and mask are then secured to the absorbing frame which is aligned directly with the boresight from transmit to receive antennas. The antennas are placed such that the wafer is illuminated by a plane wave, being in the far field of the two antennas. In the absence of the mask and glue, the wafer has a response typical of that for a dielectric with $\varepsilon_{r}$ of 11.8, including the losses due to mismatch between the air and dielectric at these frequencies. Owing to ambiguities that may exist in the dark state of the silicon, glue and mask, we have normalized the results with respect to the dark state transmission, including the mask and glue. The wafer exhibits an insertion loss of around $2.5 \mathrm{~dB}$ at 16 $\mathrm{GHz}$, increasing to around $4 \mathrm{~dB}$ at $40 \mathrm{GHz}$. The output power from the RF source was set at about $3 \mathrm{~mW}$, although this is not critical as the results are normalized.

Figs. 4-6 show comparisons of theoretical and measured data for three different grid arrays, depicting clearly the inductive effect at lower frequencies. For all the theoretical plots we have used a single modal index of 13 (i.e., $|p|=13, q=0$ ) since only one dimensional array is considered, together with 


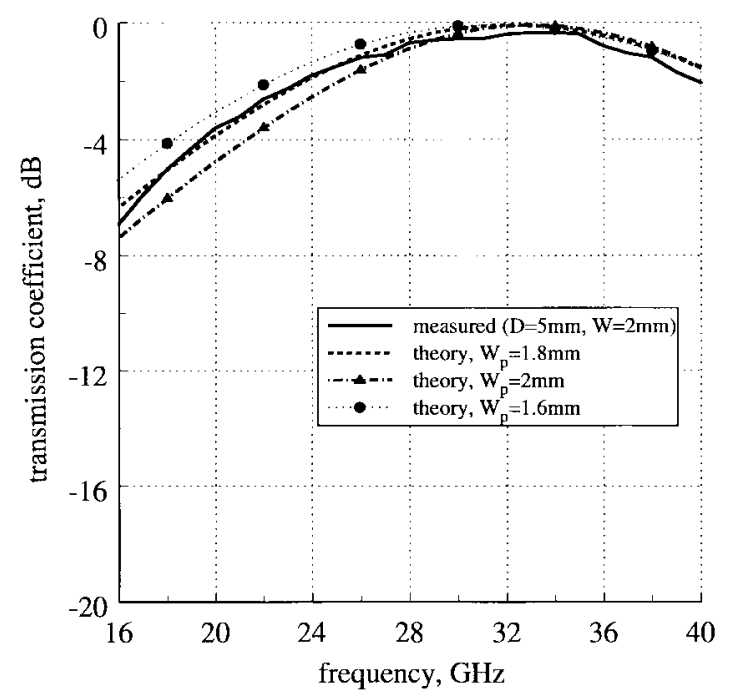

Fig. 4. Transmission response of plasma grid $D=5 \mathrm{~mm}, W=2 \mathrm{~mm}$.

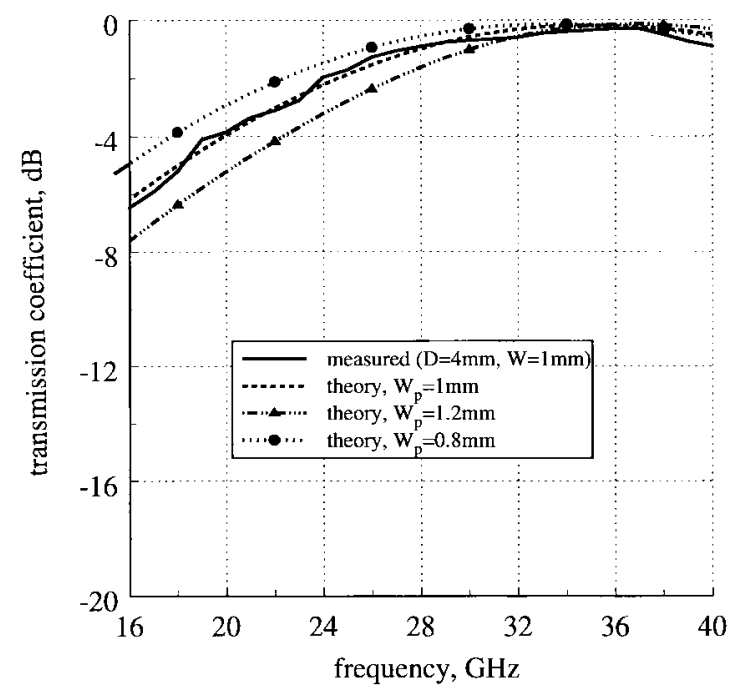

Fig. 5. Transmission response of plasma grid $D=4 \mathrm{~mm}, W=1 \mathrm{~mm}$.

four entire domain basis functions to represent the unknown current. It was assumed that the plasma penetrated half the thickness of the wafer (i.e., $S_{p}=165 \mu \mathrm{m}$ ) and that the remaining $165 \mu \mathrm{m}$ was pure silicon with $\varepsilon_{u}=11.8$. In all cases the carrier density was taken to be $10^{17} \mathrm{~cm}^{-3}$. It can be seen that for all cases considered here there is a discrepancy between $W$ and $W_{p}$, where $W_{p}$ is the width of the plasma strips needed to closely match the measured results with the theory. The combination of $N, W_{p}, S_{p}$, and $S_{d}$ was deduced from the agreement between theoretical and measured results but the photoinduced plasma may be influenced by the optical absorption depth and/or diffusion length, that may infer a lower plasma density. Although the absorption depth in the spectrum of our light source is less than $100 \mu \mathrm{m}$, the chosen $S_{p}$ may be viewed as an effective penetration depth; a combination of optical absorption depth and carrier diffusion. In addition, our model allows only canonical, three-dimensional blocks to represent the plasma elements. The incident photons will be diffracted to a certain extent by the black regions of the

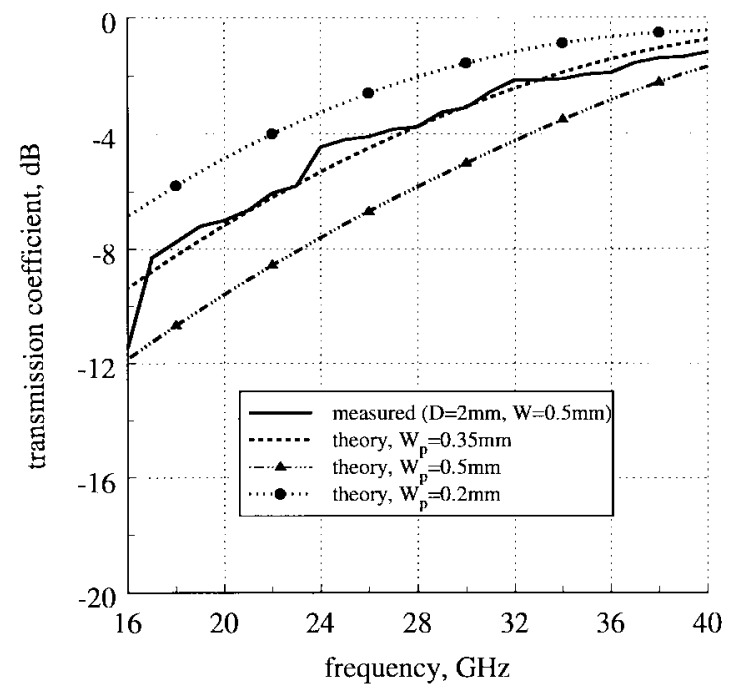

Fig. 6. Transmission response of plasma grid $D=2 \mathrm{~mm}, W=0.5 \mathrm{~mm}$.

mask, and will, therefore, not all penetrate normally, and some will diffuse to outside the extent of the transparent region of the mask. Further, the number of photon-electron collisions taking place make it highly probable that even those photons that are normally incident will not continue in this direction for long. The random nature of the collisions, the incident light intensity and carrier lifetime will all also have a bearing on the exact dimensions and extent of the plasma generated. Nevertheless, we have achieved good agreement with the measured data using the assumptions already stated. Namely: $N=10^{17} \mathrm{~cm}^{-3}, S_{p}=165 \mu \mathrm{m}$ and $W_{p}$ as stated in the legend of Figs. 4-6. We have also plotted theoretical curves for values of $W_{p}$ slightly above and below the optimum value and presented them on the respective graphs. The effect of the change in strip width is not surprising and is about the same as would be observed for a conventional copper inductive grid. The crucial point to note from these results comes from Fig. 4, where the periodicity is just large enough $(5 \mathrm{~mm})$ to reveal the effects of the grating lobe region at around $40 \mathrm{GHz}$, that further verifies that we have produced a photoinduced plasma inductive grid in the silicon slab.

\section{Influence of Plasma Density on TRANSMITTED AND DisSIPATED POWER}

The level of generated electron-hole pairs or plasma density $N$ is a measure of how metallic the silicon has become when illuminated. We have performed a study to determine the effect of $N$ on the transmitted power and the power dissipated in the plasma region, for various combinations of plasma and dielectric thicknesses. The mathematical model handles discrete layers of varying, definable properties and in order to match our measured results we adopted the scheme that the plasma penetrated halfway through the wafers, i.e., $S_{d}=S_{p}=$ $165 \mu \mathrm{m}$. Fig. 7(a) and (b) shows theoretical results for transmission coefficient and dissipated power (within the wafer). The dimensions of the grid are $D=5 \mathrm{~mm}$ and $W=2 \mathrm{~mm}$.

From these graphs it is clear that when $N=10^{20} \mathrm{~cm}^{-3}$ the plasma is almost totally metallic. There is little loss at 34 


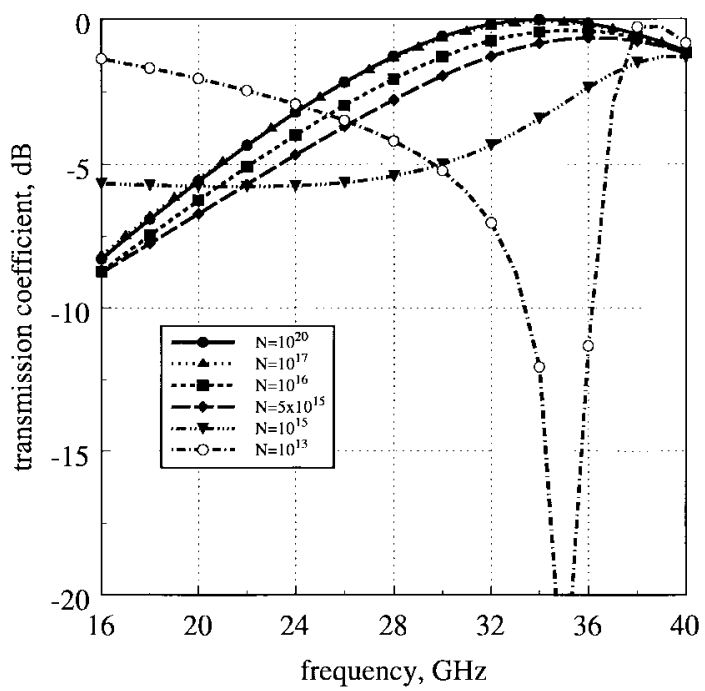

(a)

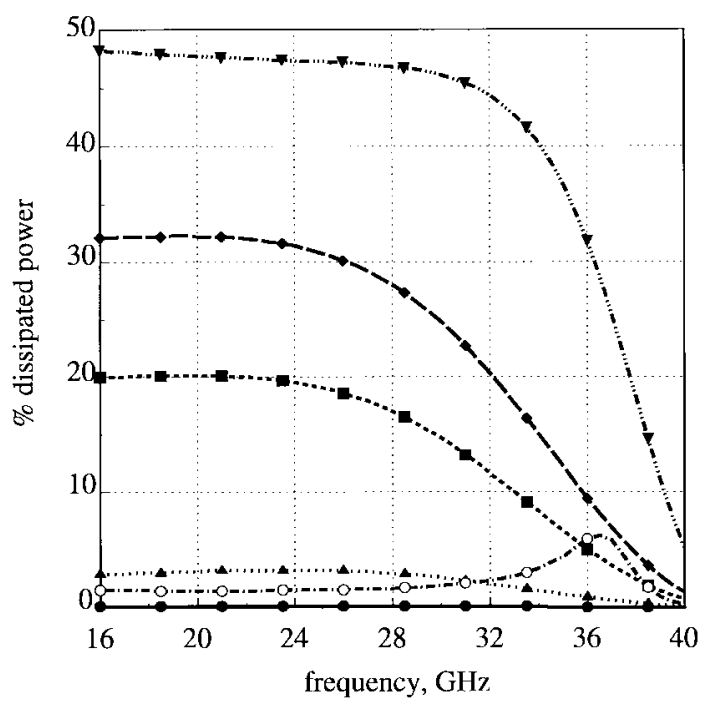

(b)

Fig. 7. Effect of varying $N$, with $S_{p}=S_{d}=165 \mu \mathrm{m}$. (a) Transmission coefficient. (b) Dissipated power.

$\mathrm{GHz}$ and more significantly, virtually no dissipative loss. We assumed in these calculations that $N=10^{17} \mathrm{~cm}^{-3}$ and this has been born out by the results above because the transmission coefficient is coincident with that when the plasma is nearly metallic. The interesting aspect of these results is the effect on dissipated loss when the silicon is in transition from dielectric to metallic. When $N=10^{13} \mathrm{~cm}^{-3}$ there is very little dissipative loss because the region defined as plasma is essentially silicon but the transmission properties seem to indicate that the whole structure acts as a dielectric grating with a deep null which is dependant on the periodicity. As $N$ is increased, the plasma becomes very lossy and affects the transmission properties of the wafer to such an extent that, for instance, at $24 \mathrm{GHz}$ with $N=10^{15} \mathrm{~cm}^{-3}, 48 \%$ of the incident power is dissipated in the silicon. This decreases with frequency for a given $N$ and becomes close to zero across the range when the wafer is metallic.

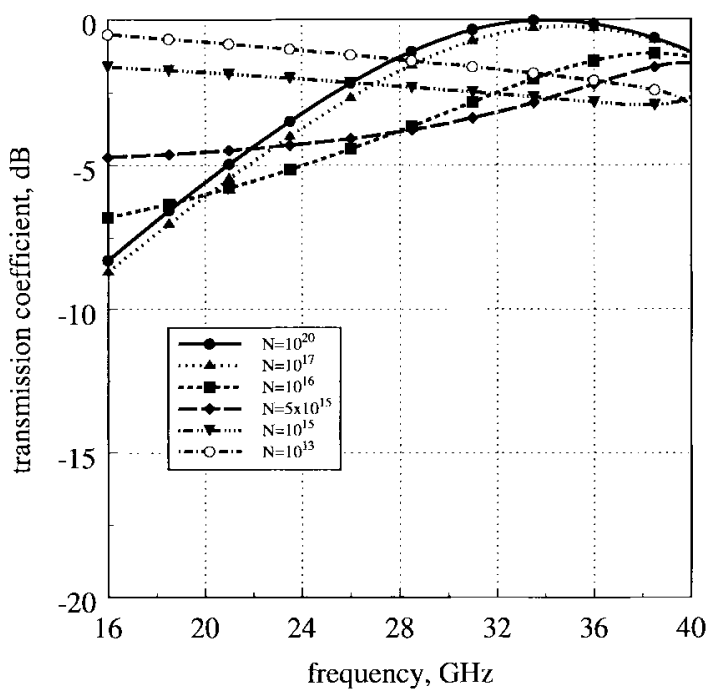

(a)

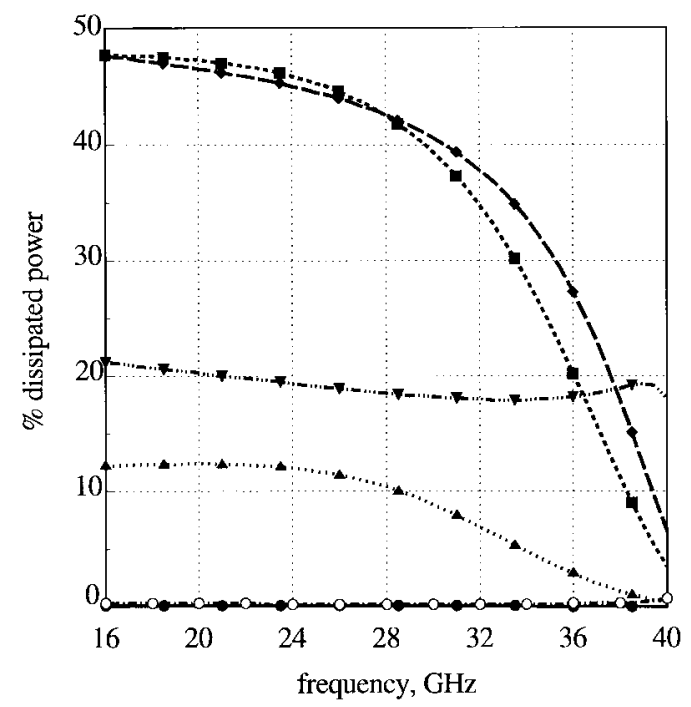

(b)

Fig. 8. Effect of varying $N$, with $S_{p}=30 \mu \mathrm{m}, S_{d}=165 \mu \mathrm{m}$. (a) Transmission coefficient. (b) Dissipated power.

For comparison purposes we looked at the situation where $S_{p}=30 \mu \mathrm{m}$ and $S_{d}=165 \mu \mathrm{m}$ to establish the influence of a thinner plasma layer on the same dielectric. Fig. 8(a) and (b) shows the theoretical results for transmitted and dissipated power. The thinner plasma layer has a clear effect on all the transmission responses apart from that when $N=10^{20} \mathrm{~cm}^{-3}$. This is due to the fact that at this density the thickness has little effect on performance because the plasma is metallic at any thickness. At lower densities the effect is greater because the plasma is in an indistinct region between dielectric and conductor. Our choice of $N=10^{17} \mathrm{~cm}^{-3}$ exhibits a bit more dissipative loss at this thickness and the transmission coefficient is reasonably stable although the centre frequency of the passband has moved up in frequency by about $1 \mathrm{GHz}$. Whereas it was coincident with the transmission coefficient for $N=10^{20} \mathrm{~cm}^{-3}$ it has now become slightly lossy at the passband and shifted up in frequency. This demonstrates that $10^{17} \mathrm{~cm}^{-3}$ does not represent pure metallic behavior. 


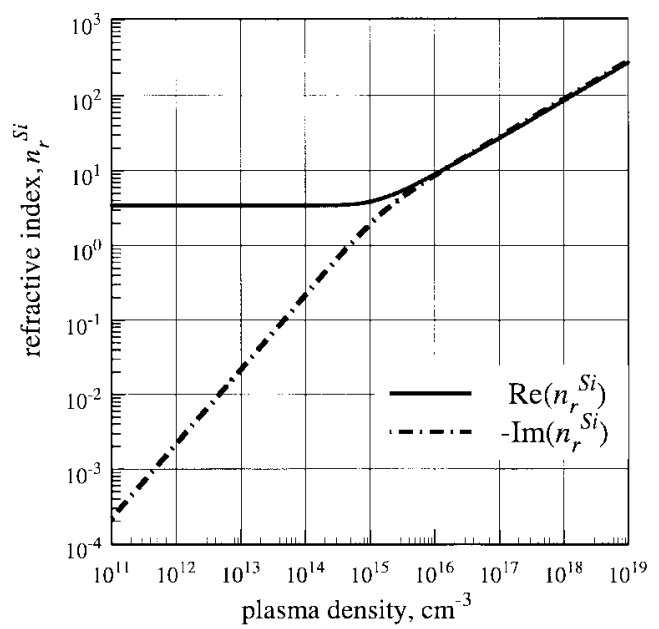

Fig. 9. Refractive index of silicon as a function of plasma concentration.

In fact the thickness of the dielectric behind the plasma will largely define the frequency of the passband, provided $N$ is large enough, if the periodicity and the width are fixed. The assumption of $S_{d}$ to be $165 \mu \mathrm{m}$ has been established to be valid because provided the plasma density is high enough then $S_{p}$ is not critical to the frequency of operation. The plasma thickness becomes crucial, however, when the plasma is between dielectric and metallic and is essentially a layer of lossy material. In practice there would not be clearly defined demarcation between dielectric and plasma but almost certainly a "plasma gradient" from one side of the wafer to the other.

An important point to make is recognizing the impact of the grating lobes on the level of dissipated power. In Figs. 7 and 8 , the dissipated power tends to decrease as the frequency rises toward $40 \mathrm{GHz}$. This is due to the fact the grating lobes have not encroached into our frequency range and is independent of the value of $N$.

\section{CONCLUSION}

We have demonstrated a basic technique for optically generating arrays of periodic grids as a pseudometallic, electron-hole plasma region within a semiconductor wafer. The wafers used were high resistivity silicon to give dielectriclike dark behavior, in order to contrast with the generated plasma. The theory of the work is based on the characterization of the plasma by means of its surface impedance, which in turn is dependent on the complex permitivitty. We have demonstrated theoretically, and verified experimentally, the generation of grid arrays of several different dimensions and shown that the array performance is largely determined by the density of the plasma, $N$. Plasma densities of $10^{17} \mathrm{~cm}^{-3}$ are necessary to achieve the near metallic state required to resonate as a conventional conductor. At lower densities the silicon becomes, essentially, a lossy medium in which up to $48 \%$ of incident power is dissipated. Tied in with this we have highlighted the effects of the plasma thickness and shown that this is not critical to performance provided the density is high enough, although the thickness of any dielectric support layers needs to be considered as this will primarily determine passband frequency for a given plasma. Results have been presented in the frequency range $16-40 \mathrm{GHz}$ to confirm that grids have been optically cast onto the wafers using negative image masks of the desired pattern.

This work forms the basis of a longer term program to develop a fully integrated technique for the formation of resonant periodic arrays of plasma, within silicon, which may be in the form of cells of discrete elements and comparable in dynamic characteristics to a conventional FSS.

\section{APPENDIX \\ Complex Relative Permittivity of Si}

The semiconductor complex relative permittivity containing electron-hole pairs is expressed as a sum of two, electrons $(e)$ and holes $(h)$ dependent terms

$$
\varepsilon_{r}^{\mathrm{Si}}=\varepsilon_{u}-\sum_{i=e, h} \frac{\omega_{p i}^{2}}{(2 \pi f)^{2}+\nu_{i}^{2}}\left(1+j \frac{\nu_{i}}{2 \pi f}\right)
$$

where $\omega_{p i}^{2}=\left(N q^{2} / \varepsilon_{o} m_{i}\right)$ is the plasma's angular frequency, $\varepsilon_{u}$ is the dark dielectric constant, $\nu_{i}$ is the collision angular frequency, $m_{i}$ is the effective mass of the carrier, $q$ is the electronic charge and $\varepsilon_{o}$ is the permittivity of freespace. For the silicon substrate the following values have been used in the computer model: $\varepsilon_{u}=11.8, \nu_{e}=4.53 \times 10^{12} \mathrm{~s}^{-1}, \nu_{h}=$ $7.71 \times 10^{12} \mathrm{~s}^{-1}, m_{e}=0.259 m_{o}, m_{h}=0.38 m_{o}, m_{o}$ is the free electronic mass.

Fig. 9 shows the refractive index of silicon versus $N$ at $40 \mathrm{GHz}$. Typically, at low plasma concentration the real part initially remains almost constant and then increases in such a way that (near $1.0 \times 10^{16} \mathrm{~cm}^{-3}$ ) it coalesces with the imaginary part reaching a value of about 10 . Subsequently, they both increase with the same rate and have similar values. They reach a value of about 1000 at a concentration of $1.0 \times 10^{20} \mathrm{~cm}^{-3}$, at which the semiconductor has reached its near metallic state.

\section{REFERENCES}

[1] J. C. Vardaxoglou, "Optical switching of frequency selective surface bandpass response," Electron. Lett., vol. 32, pp. 2345-2346, 1996.

[2] M. Matsumoto, M. Tsutsumi, and N. Kumagai, "Radiation of millimeter waves from a leaky dielectric waveguide with a light induced grating layer," IEEE Trans. Microwave Theory Tech., vol. MTT-35, pp. 1033-1042, Nov. 1987.

[3] A. Alphones and M. Tsutsumi, "Leaky wave radiation from a periodically photoexcited semiconductor slab waveguide," IEEE Trans. Microwave Theory Tech., vol. 43, pp. 2435-2441, Oct. 1995.

[4] _ "Optically controlled Bragg reflection characteristic of millimeter waves in a corrugated dielectric waveguide," Microwave Opt. Tech. Lett., vol. 17, no. 6, pp. 352-357, 1998.

[5] K. Nishimura and M. Tsutsumi, "Scattering of millimeter waves by metallic strip gratings on an optically plasma-induced semiconductor slab," IEEE Trans. Microwave Theory Tech., vol. 44, pp. 2231-2237, Dec. 1996.

[6] W. Platte, "Periodic-structure photoexcitation of a silicon coplanar waveguide for selective optoelectronic microwave control," IEEE Trans. Microwave Theory Tech., vol. 38, pp. 638-646, May 1990.

[7] D. Liu, D. Charette, M. Bergeron, H. Karwacki, S. Adams, B. Lanning, and F. Kustas, "Structurally embedded photoconductive silicon bowtie antenna," IEEE Photon. Technol. Lett., vol. 10, pp. 716-718, May 1998. 
[8] J. C. Vardaxoglou, "Optical switching and generation of periodic arrays and FSS," invited presentation, in Proc. IEEE Int. Conf. Electromagnetics in Advanced Applications, ICEAA, Torino, Italy, Sept. 1997, pp. $51-56$.

[9] J. C. Vardaxoglou, P. K. Lau, and M. J. Kearney, "Frequency selective surface from optically excited semiconductor on a substrate," Electron. Lett., vol. 34, pp. 570-571, 1998.

[10] J. C. Vardaxoglou, Frequency Selective Surfaces: Analysis and Design. New York: Wiley, 1997, ch. 2.

[11] C. A. Balanis, Advanced Engineering Electromagnetics. New York: Wiley, 1989, Sec. 2.8 .

[12] R. E. Colin, Foundations for Microwave Engineering, 2nd ed. New York: McGraw Hill, 1992, Sec 2.9.

[13] R. Orta, P. Savi, and R. Tascone, "Recent developments in frequency selective surfaces," in JINA'92, Nice, France, 1992, pp. 223-232.
J. C. Vardaxoglou (M'88) received the B.Sc. degree in mathematics (mathematical physics) in 1981, and the Ph.D. degree in 1985, both from the University of Kent, Canterbury, U.K.

$\mathrm{He}$ was appointed Lecturer in Communications in the Department of Electronic and Electrical Engineering at Loughborough University of Technology, Loughborough, U.K., in January 1988, and he was promoted to the position of Senior Lecturer in January 1992. In 1998, he was appointed Professor of Wireless Communications. He established the Antennas and Microwaves Research at Loughborough University and heads the Centre for Mobile Communications Research (CMCR). He has been active in the area of electromagnetic modeling and applications of frequency selective surfaces. His current research interests include wireless communication networks, array antennas, FSS, radomes, leaky-wave resonant antennas, optical control of microwaves and devices, periodic surfaces and PBG materials, and mobile telephone antennas. His contribution to the CMCR lies in the analysis and design of small bifilar and quadrifilar antennas for mobile telephony. He has served as a consultant to various industries in the U.K. and abroad. He has published over 100 refereed journals and conference proceedings papers and has written a research monograph on frequency selective surfaces.

M. J. Kearney was born in Crawley, U.K., in 1962. He received the B.A degree in physics from the University of Oxford, Oxford, U.K., in 1985, and the Ph.D. degree in physics (theoretical solid state) from the University of Warwick, U.K., in 1988.

Between 1988 and 1995, he worked for the GEC-Marconi Hirst Research Centre, Wembley, U.K., becoming manager of their Long Range Research Laboratory in 1994. In 1995, he was appointed Professor of Electronic Device Engineering in the Department of Electronic and Electrical Engineering, Loughborough University, Loughborough, U.K. In 1997, he was appointed Head of Department. The author or co-author of over 60 technical articles, he has worked on topics ranging from high-frequency GaAs devices for radar applications, through fundamental studies of SiGe heterostructures for highspeed electronics, to signal processing in radar, communications, and imaging systems. 